\title{
Born-Oppenheimer approximation in EFT and quarkonium hybrids
}

\author{
Jaume Tarrús Castellà ${ }^{1, a}$ \\ ${ }^{1}$ Physik-Department, Technische Universität München, \\ James-Franck-Str. 1, 85748 Garching, Germany
}

\begin{abstract}
We report on the results of [1] for the calculations of quarkonium hybrids. We have developed and Effective Field Theory (EFT) for quarkonium hybrids that systematically incorporates an expansion with respect to the adiabatic limit. We matched the potentials in our EFT to the static energies computed on the lattice. We discuss our results and compare them with direct lattice calculations and possible experimental candidates.
\end{abstract}

\section{Introduction}

During the past decade, experimental observations have revealed the existence of a large number of unexpected states close to or above the open favor thresholds in the quarkonium spectrum, that do not fit standard potential interaction picture [2]. It began with the discovery of the $X(3872)$ by the Belle Collaboration in September 2003 [3]. This charmonium state has decays that severely violate isospin symmetry, disfavoring its identification as conventional charmonium. It continued with the discovery of the $Y(4260)$ by the BABAR Collaboration in July 2005 [4]. This charmonium state has $J^{P C}$ quantum numbers $1^{--}$, but it is produced very weakly in $e^{+} e^{-}$annihilation. Over the following years many new states were observed, most of which display special features that single them out as "exotic" states, culminating recently with the observation of a charmonium-pentaquark state at LHCb [5]. They have been observed at accelerator experiments like BES (IHEP, China), CDF and D0 at Fermilab (USA), CLEO (Cornell,USA) BaBar (SLAC, USA), Belle (KEK, Japan) and are now being continuously discovered at the upgraded version of BESIII in China, and at LHC experiments at CERN and soon again at Belle II in Japan, see $[6,7]$ for a recent review.

Exotic quarkonia are extremely interesting since they are candidates for nontraditional hadronic states, that is states that cannot be classified as mesons or baryons. Many phenomenological models for exotics have been proposed which can be loosely classified into two categories, those containing an excited gluonic state and the ones containing four constituent quarks. The latter can be further subdivided corresponding to different spatial arrangements of the four constituent quarks: meson molecules, diquarkonium, compact tetraquarks and hadro-quarkonium, to name a few examples.

In the present work we examine the possibility that some of the exotic quarkonium states are quarkonium hybrids. Such stats consist of a heavy quark and an antiquark in a color octet configuration together with a gluonic excitation. Quarkonium hybrids are characterized by the vast dynamical

ae-mail: jaume.tarrus@tum.de 
difference between the slow and massive heavy quarks with the fast and massless gluons. Since the gluons move much faster than the heavy quarks it is expected that the gluonic fields adapt nearly instantaneously to the changes in the heavy quarks separation.

This physical situation is analogous to the one in molecules, where the nuclei play the role of the heavy degrees of freedom and the electrons that of the light degrees of freedom. To solve the Schrödinger equation for these systems the Born-Oppenheimer (BO) approximation was developed. In the BO approximation the Schrödinger equation is solved in a two step process. First one solves the equation assuming the nuclei are static. The corresponding eigenenergies are the so-called electronic static energies. In a second step the total wavefunction of the system is expanded in the electronic static eigenfunctions. Introducing this expansion into to the Schrödinger equation results in an equation for the nuclei in the background potential created by the electronic static energies.

\section{EFT for quarkonium hybrids}

A nonrelativistic heavy-quark antiquark bound state is characterized by three well separated scales: the heavy quark mass $M$ (hard scale), the relative momentum $M w$ (soft scale), where $w$ is the heavy quark relative velocity, and the binding energy $M w^{2}$ (ultrasoft scale). Furthermore there is the scale associated with non-perturbative physics, $\Lambda_{\mathrm{QCD}}$. Restricting ourselves to the case in which $M w \gg$ $\Lambda_{\mathrm{QCD}}$, one can construct weakly-coupled pNRQCD $[8,9]$ to describe heavy-quark antiquark bound states, which at leading order in $1 / M$ and at $O(r)$ in the multipole expansion reads as

$$
\begin{aligned}
\mathcal{L}_{\mathrm{pNRQCD}}= & \int d^{3} r \operatorname{Tr}\left[S^{\dagger}\left(i \partial_{0}-h_{s}\right) S+O^{\dagger}\left(i D_{0}-h_{o}\right) O\right]-\frac{1}{4} G_{\mu \nu}^{a} G^{\mu \nu a} \\
& +g V_{A}(r) \operatorname{Tr}\left[O^{\dagger} \boldsymbol{r} \cdot \boldsymbol{E} S+S^{\dagger} \boldsymbol{r} \cdot \boldsymbol{E} O\right]+\frac{g}{2} V_{B}(r) \operatorname{Tr}\left[O^{\dagger} \boldsymbol{r} \cdot \boldsymbol{E} O+O^{\dagger} \boldsymbol{O} \cdot \boldsymbol{E}\right],
\end{aligned}
$$

where $S$ and $O$ are the quark singlet and octet fields respectively normalized with respect to color and should be understood as depending on $t, \boldsymbol{r}$ the relative coordinate and $\boldsymbol{R}$ the center of mass of the heavy quarks. All the fields of the light degrees of freedom in Eq. (1) are evaluated at $\boldsymbol{R}$ and $t$; in particular, $G^{\mu v a} \equiv G^{\mu v a}(\boldsymbol{R}, t)$ and $i D_{0} O \equiv \partial_{0} O-g\left[A_{0}(\boldsymbol{R}, t), O\right]$. We will not consider light-quark contributions in the present work. The singlet and octet Hamiltonian densities read

$$
h_{s}=-\frac{\boldsymbol{\nabla}_{r}^{2}}{M}+V_{s}(r), \quad h_{o}=-\frac{\boldsymbol{\nabla}_{r}^{2}}{M}+V_{o}(r) .
$$

The pNRQCD Lagrangian of Eq. 1, does not only describe ultrasoft degrees of freedom but also degrees of freedom associated with the scale $\Lambda_{Q C D}$ which are still dynamical. If we are only interested in the lower lying states, it is convenient to integrate out the scale $\Lambda_{Q C D}$ in order to obtain a suitable EFT for ultrasoft degrees of freedom only. We are going to do so in a procedure analogous to the one used in the BO approximation: first we are going to determine the eigenstates of the leading order Hamiltonian of pNRQCD and expand the Lagrangian into the basis they define. In a second step we formally integrate out the gluonic degrees of freedom at the $\Lambda_{Q C D}$ scale. Note that since we are interested in quarkonium hybrids we are going to only consider the octet sector.

To simplify the analysis let us assume that the following scale hierarchy holds true: $M w \gg$ $\Lambda_{Q C D} \gg M w^{2}$. The Hamiltonian density corresponding to the gluonic degrees of freedom at leading order in $1 / M$ and in the multipole expansion is

$$
h_{0}(\boldsymbol{R})=\frac{1}{2}\left(\boldsymbol{E}^{a} \boldsymbol{E}^{a}+\boldsymbol{B}^{a} \boldsymbol{B}^{a}\right)
$$


The symmetry group of (3) is $O(3) \times C$. The color octet glue operators $G_{i K}^{a}(\boldsymbol{R})$, the so-called gluelumps, generate the eigenstates of $h_{0}(\boldsymbol{R})$

$$
\hat{h}_{0}(\boldsymbol{R}) G_{i \kappa}^{a}(\boldsymbol{R})|0\rangle=\Lambda_{\kappa} G_{i \kappa}^{a}(\boldsymbol{R})|0\rangle,
$$

and form a basis of octet glue operators in pNRQCD. The gluelump operators are labeled by the $\kappa=J^{P C}$ quantum numbers, and $i$ labels the different components for each $J^{P C}$ representation.

Let us introduce the states

$$
|\kappa\rangle=O^{a \dagger}(\boldsymbol{r}, \boldsymbol{R}) G_{i \kappa}^{a}(\boldsymbol{R})|0\rangle,
$$

which are eigenstates of the octet sector of the pNRQCD Hamiltonian at leading order in the multipole expansion with eigenvalue $h_{o}+\Lambda_{\kappa}$. We can now expand the octet sector of the pNRQCD Lagrangian using the basis of gluelump operators by projecting the Lagrangian of Eq. (1) into the Fock-subspace spanned by

$$
\int d^{3} r d^{3} R \sum_{\kappa}|\kappa\rangle \Psi_{i \kappa}(t, \boldsymbol{r}, \boldsymbol{R}) .
$$

Using Eq. (6) and integrating out the gluonic degrees of freedom of energy of the order $\Lambda_{Q C D}$ one can arrive at the BO EFT Lagrangian that describes the heavy-quark pair physics at the ultrasoft scale. Since we are interested in bound states we are going to ignore transitions between states with different $\kappa$ and decays into singlet states. Up to next-to-leading order in the multipole expansion the Lagrangian reads

$$
\mathcal{L}_{B O}^{o}=\int d^{3} r \sum_{\kappa} \Psi_{i \kappa}^{\dagger}(t, \boldsymbol{r}, \boldsymbol{R})\left[\left(i \partial_{t}-h_{o}-\Lambda_{\kappa}\right) \delta^{i j}-\sum_{\lambda} P_{\kappa \lambda}^{i} b_{\kappa \lambda} r^{2} P_{\kappa \lambda}^{j}+\cdots\right] \Psi_{j \kappa}(t, \boldsymbol{r}, \boldsymbol{R}),
$$

where $P_{\kappa \lambda}^{i}$ are projectors into the heavy quark axis of the gluelump operator. There is a projector for each $-|j| \leq \lambda \leq|j|$. These projectors select different polarizations of the wavefunction $\Psi_{i k}$. For example, in the case of $J=1$ the projectors are given by

$$
\begin{aligned}
P_{10}^{l} & =\hat{r}^{l}, \\
P_{1 \pm 1}^{l} & =\left(\hat{\theta}^{l} \pm i \hat{\phi}^{l}\right) / \sqrt{2},
\end{aligned}
$$

with

$$
\begin{aligned}
& \hat{r}=(\sin (\theta) \cos (\phi), \sin (\theta) \sin (\phi), \cos (\theta))^{T}, \\
& \hat{\theta}=(\cos (\theta) \cos (\phi), \cos (\theta) \sin (\phi),-\sin (\theta))^{T}, \\
& \hat{\phi}=(-\sin (\phi), \cos (\phi), 0)^{T} .
\end{aligned}
$$

For higher $J$ the projector operators can be build by multiplying $|j|$ powers of (8) and (9) with appropriate symmetrization of the indices. In Eq. (7) the $P_{k \lambda}^{i} b_{\kappa \lambda} r^{2} P_{k \lambda}^{j}$ term corresponds to the next-to-leading order in the multipole expansion and the dots stand for higher order corrections.

The specific value of the next-to-leading order term depends on non-perturbative physics and is unknown, however some of its characteristics can be determined from general grounds. This term has its origin in the chromo-electric dipole couplings of the octet field in Eq. (1), which couple the gluelump operator $G_{i \kappa}^{a}$ to the octet field giving corrections to the energy of the system. The 
$r^{2}$ dependence arises from the necessity of having at least two chromo-electric dipolar couplings in order to conserve the $J^{P C}$ quantum numbers of $G_{i k}^{a}$. Moreover, for finite separation between the heavyquark pair, the energy eigenstates of the system must be organized in representations of the cylindrical symmetry group $D_{\infty h}$, with parity replaced by $C P$. The representations of $D_{\infty h}$ are raditionally denoted by $\Lambda=|\lambda|$ and conventionally labeled by $\Sigma, \Pi, \Delta, \cdots$ referring to $\Lambda=0,1,2, \cdots$; the eigenvalues \pm 1 of the $C P$ operator, denoted by $g=+1$ and $u=-1$; and for the $\Sigma$ state, there is a symmetry under reflection in any plane passing through the axis $\hat{r}$, the eigenvalues of the corresponding symmetry operator being \pm 1 . Such representations can be obtained by projecting the gluelump operator in Eq. (5) into the heavy quark axis giving rise to the $P_{k \lambda}^{i}$ projectors in Eq. (7). Cylindrical symmetry and charge conjugation also imply $b_{\kappa \lambda}=b_{\kappa-\lambda}$.

Defining the projected wavefunction as

$$
\Psi_{\kappa \lambda}=P_{\kappa \lambda}^{i} \Psi_{i \kappa}, \quad \Psi_{i \kappa}=\sum_{\lambda} P_{i \kappa \lambda} \Psi_{\kappa \lambda},
$$

in Eq. (7) we obtain

$$
\mathcal{L}_{B O}^{o}=\int d^{3} r \sum_{\kappa} \sum_{\lambda \lambda^{\prime}} \Psi_{k \lambda}^{\dagger}(t, \boldsymbol{r}, \boldsymbol{R})\left\{\left[i \partial_{t}-V_{o}(r)-\Lambda_{\kappa}-b_{\kappa \lambda} r^{2}+\cdots\right] \delta_{\lambda \lambda^{\prime}}+P_{\kappa \lambda}^{i} \frac{\nabla_{r}^{2}}{M} P_{i \kappa \lambda^{\prime}}\right\} \Psi_{\kappa \lambda^{\prime}}(t, \boldsymbol{r}, \boldsymbol{R}) .
$$

The last term can be splitted into a kinetic operator acting onto the heavy-quark field and a nonadiabatic coupling

$$
P_{\kappa \lambda}^{i} \frac{\nabla_{r}^{2}}{M} P_{i \kappa \lambda^{\prime}}=\frac{\nabla_{r}^{2}}{M}+C_{\kappa \lambda \lambda^{\prime}},
$$

with

$$
C_{\kappa \lambda \lambda^{\prime}}=P_{\kappa \lambda}^{i}\left[\frac{\nabla_{r}^{2}}{M}, P_{i \kappa \lambda^{\prime}}\right]
$$

being the nonadiabatic coupling analogous to one encountered in diatomic molecules. The equation of motion for the fields $\Psi_{\kappa \lambda}(t, \boldsymbol{r}, \boldsymbol{R})$ that follow from the Euler-Lagrange equation is nothing else than a set of coupled Schrödinger equations

$$
i \partial_{t} \Psi_{\kappa \lambda}(t, \boldsymbol{r}, \boldsymbol{R})=\left[\left(-\frac{\boldsymbol{\nabla}_{r}^{2}}{M}+V_{o}(r)+\Lambda_{\kappa}+b_{\kappa \lambda} r^{2}+\ldots\right) \delta_{\lambda \lambda^{\prime}}-\sum_{\lambda^{\prime}} C_{\kappa \lambda \lambda^{\prime}}\right] \Psi_{\kappa \lambda^{\prime}}(t, \boldsymbol{r}, \boldsymbol{R}) .
$$

It is important to note that the nonadiabatic coupling mixes states generated by the same gluelump (i.e. with the same $\kappa$ quantum numbers) and corresponding to different projections onto the heavy quarkantiquark axis (i.e. different $\lambda$ ). In the short distance limit, where the $b_{\kappa \lambda} r^{2}$ term can be neglected, the potentials corresponding to the mixed states are degenerate. Due to this quasi-degeneracy of the potentials we find more convenient to consider the coupled Schrödinger equations instead of treating the mixing terms as a perturbation.

Solving the coupled Schrödinger equations one obtains the eigenvalues $\mathcal{E}_{N}$ that give the masses $M_{N}$ of the states as

$$
M_{N}=2 M+\mathcal{E}_{N}
$$

\section{Matching of the potential to the lattice static energies}

Let $\left|\boldsymbol{x}_{1}, \boldsymbol{x}_{2}, \underline{n}\right\rangle^{(0)}$ be a gauge-invariant eigenstate of the NRQCD Lagrangian with a heavy quarkantiquark pair in the static limit. $\boldsymbol{x}_{1}$ and $\boldsymbol{x}_{2}$ are the heavy quark positions and $n$ labels the representations of $D_{\infty h}$, the (0) superscript denotes the static limit for the heavy quarks (see $[1,10]$ for further 
details). Then

$$
H_{N R Q C D}^{(0)}\left|\boldsymbol{x}_{1}, \boldsymbol{x}_{2}, \underline{n}\right\rangle^{(0)}=E_{n}^{(0)}(r)\left|\boldsymbol{x}_{1}, \boldsymbol{x}_{2}, \underline{n}\right\rangle^{(0)}, \quad r=\left|x_{1}+\boldsymbol{x}_{2}\right| .
$$

The energy eigenvalues, $E_{n}^{(0)}$, are the so-called static energies. The set of $\left|\boldsymbol{x}_{1}, \boldsymbol{x}_{2}, \underline{n}\right\rangle^{(0)}$ states form a compatible basis, therefore any state can be expressed in this basis

$$
\left|X_{n}\right\rangle=\sum_{n^{\prime}} c_{n^{\prime}}\left|\boldsymbol{x}_{1}, \boldsymbol{x}_{2}, \underline{n}^{\prime}\right\rangle^{(0)}
$$

It follows that

$$
\left\langle X_{n}, T / 2 \mid X_{n},-T / 2\right\rangle=\mathcal{N}\left(\left|c_{n}\right|^{2} e^{-i E_{n}^{(0)} t}+\left|c_{n^{\prime}}\right|^{2} e^{-i E_{n^{\prime}}^{(0)} t}+\ldots\right) .
$$

In euclidean time, for large $t$, the correlator is dominated by the lowest energy eigenvalue, thus the static energies can be obtained even without explicitly knowing the static states

$$
E_{n}^{(0)}=\lim _{T \rightarrow \infty} \frac{i}{T} \log \left\langle X_{n}, T / 2 \mid X_{n},-T / 2\right\rangle
$$

We just need and states $\left|X_{n}\right\rangle$ with a non-vanishing overlap with the desired static state, but otherwise we are free to choose the $\left|X_{n}\right\rangle$ state. The following choice gives the static energies in terms of static Wilson loops

$$
\left|X_{n}\right\rangle=\chi\left(\boldsymbol{x}_{2}\right) \phi\left(\boldsymbol{x}_{2}, \boldsymbol{R}\right) H_{n}^{a}(\boldsymbol{R}) T^{a} \phi\left(\boldsymbol{R}, \boldsymbol{x}_{1}\right) \psi^{\dagger}\left(x_{1}\right)|v a c\rangle,
$$

where $\psi$ and $\chi$ are the heavy quark and antiquark fields, $\phi$ are Wilson lines, $|v a c\rangle$ is the NRQCD vacuum.

The static energies for heavy quark-antiquark pairs have been computed in lattice QCD by several authors. In this work we have used the latest available data sets obtained by Juge, Kuti, and Morningstar in [11, 12] and by Bali and Pineda in [13]. Static energies were obtained in quenched lattice QCD by Juge, Kuti, and Morningstar on anisotropic lattices using an improved gauge action. They extracted the static energies from Monte Carlo estimates of generalized large Wilson loops for a large set of operators projected onto the different representations of the $D_{\infty h}$ group (see Fig. 1). Lattice simulations were carried out by Bali and Pineda in [13] focusing on the short range static energies for the $\Pi_{u}$ and $\Sigma_{u}^{-}$potentials. They performed two sets of computations using a Wilson gauge action in the quenched approximation.

The states $\left|X_{n}\right\rangle$ can be multipole expanded and matched into pNRQCD states and these in turn can be matched to BOEFT states

$$
\begin{aligned}
\left|X_{n}\right\rangle & \cong\left(Z_{n}(r) O^{a \dagger}(\boldsymbol{r}, \boldsymbol{R}) H_{n}^{a}(\boldsymbol{R})+O(r)\right)|0\rangle=\left(Z_{n}(r) O^{a \dagger}(\boldsymbol{r}, \boldsymbol{R})\left(\sum_{\kappa \lambda} c_{\kappa \lambda} P_{\kappa \lambda}^{i} G_{i \kappa}^{a}(\boldsymbol{R})\right)+O(r)\right)|0\rangle \\
& \cong Z_{n}(r) \sum_{\kappa \lambda} c_{\kappa \lambda} O^{a \dagger}(\boldsymbol{r}, \boldsymbol{R}) P_{\kappa \lambda}^{i} G_{i \kappa}^{a}(\boldsymbol{R})|0\rangle_{B O}+\ldots
\end{aligned}
$$

In the second line we used that the gluelump operators form a basis of glue operators in pNQRCD. The large time log of the correlator in euclidean space of such states is going to be dominated by the gluelump with a lower mass and nonzero overlap, thus we obtain the matching between static energies and potentials in BOEFT

$$
E_{n}^{(0)}=\lim _{T->\infty} \frac{i}{T} \log \left\langle X_{n}, T / 2 \mid X_{n},-T / 2\right\rangle=V_{o}+\Lambda_{K}+b_{\kappa \lambda} r^{2}+\cdots
$$




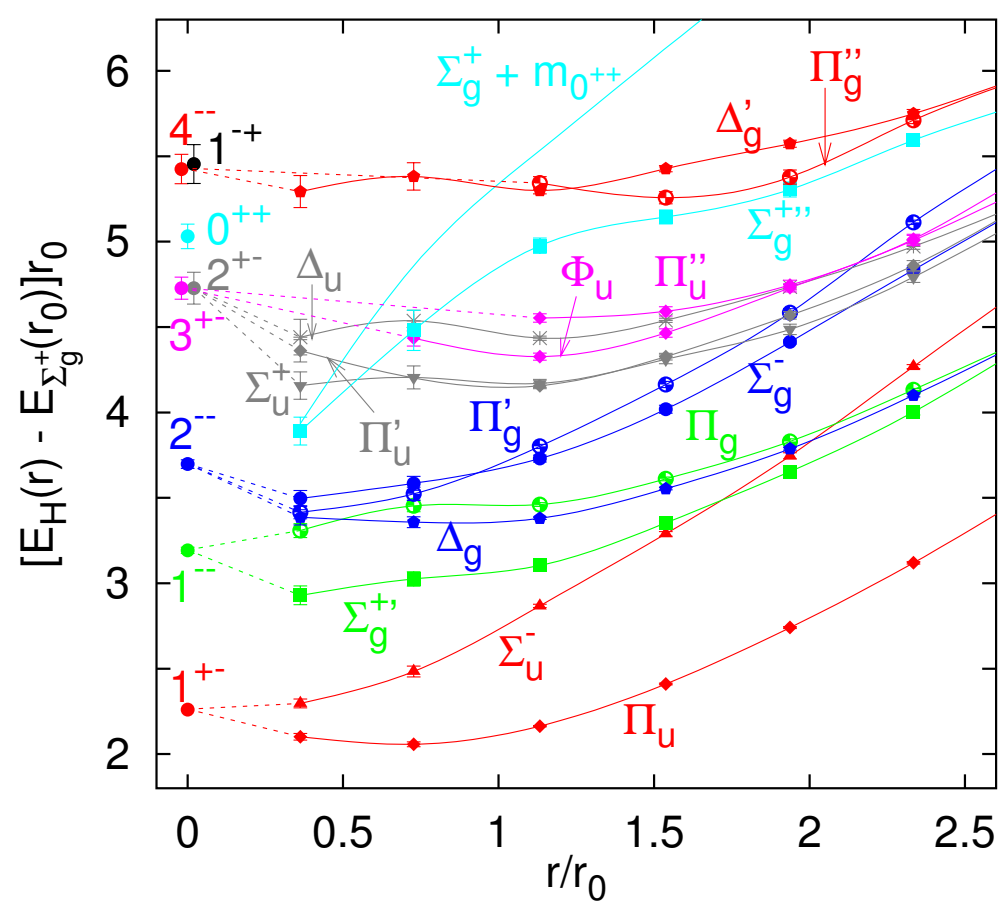

Figure 1. The lowest hybrid static energies [12] and gluelump masses [14] in units of $r_{0} \approx 0.5 \mathrm{fm}$. The absolute values have been fixed such that the ground state $\Sigma_{g}^{+}$static energy (not displayed) is zero at $r_{0}$. The behavior of the static energies at short distances becomes rather unreliable for some hybrids, especially the higher exited ones. This is largely due to the difficulty in lattice calculations to distinguish between states with the same quantum numbers, which mix. For example, the $\Sigma_{q}^{+\prime \prime}$ static energy approaches the shape corresponding to a singlet plus $0^{++}$glueball state (also displayed) instead of the $0^{++}$gluelump. This picture is taken from [13].

\section{Computation of the hybrid quarkonium masses}

We have solved the coupled Schrödinger equations that result from considering the static states generated by the lowest mass gluelump with $J^{P C}=1^{+-}$, which correspond to the representations $\Pi_{u}$ and $\Sigma_{u}^{-}$ of $D_{\infty h}$. The gluelump mass was computed on the lattice in Ref. [13] using a renormalon subtracted (RS) scheme, $\Lambda_{1^{+-}}^{R S}=0.87(15) \mathrm{GeV}$. Therefore we have used the octet potential computed in the same RS scheme.

We have constructed a potential that includes as much information as possible from the long range lattice data. This potential, which we call $V^{(0.25)}$, is defined as follows. For $r \leq 0.25 \mathrm{fm}$ we use the potential from right hand side of (24) with $b_{\kappa \lambda}$ fitted to lattice data up to $r=0.25 \mathrm{fm}$. For $r \geq 0.25 \mathrm{fm}$ we have used a fit of a function chosen to reproduce well the lattice data. To ensure a smooth transition we have imposed continuity up to first derivatives.

Like in ordinary Schrödinger equations, the wave functions can be split into an orbital and a radial part. The orbital wave functions satisfy a modified version of the defining differential equation of the spherical harmonics and are labeled by quantum numbers $l$ and $m$, which correspond to the eigenvalues of the combined angular momentum operator of the gluons and the relative quark-antiquark motion. The radial Schrödinger equation then is found to decouple into two parts: the first part is still coupled but now has only two components: 
Table 1. Hybrid energies obtained from solving the Schrödinger equation for the $V^{(0.25)}$ potentials. All values are given in units of $\mathrm{GeV}$. The primes on the multiplets indicate the first excited state of that multiplet. $J^{P C}$ quantum numbers of the hybrid states are obtained combining the angular momentum $l$ with the spin state. $\left\langle E_{k}\right\rangle$ is the mean value of the kinetic energy. $P_{\Pi}$ is a measure of the probability to find the hybrid in a $\Pi_{u}$ configuration, thus it gives a measure of the mixing effects.

\begin{tabular}{|c|c|c|c|c|c|c|c|c|c|c|c|c|c|c|}
\hline \multirow{2}{*}{ Multiplet } & \multirow{2}{*}{$l$} & \multirow{2}{*}{$J^{P C}$} & \multicolumn{4}{|c|}{$c c$} & \multicolumn{4}{|c|}{$b c$} & \multicolumn{4}{|c|}{$b b$} \\
\hline & & & $m_{H}$ & $\langle 1 / r\rangle$ & $\left\langle E_{k}\right\rangle$ & $P_{\Pi}$ & $m_{H}$ & $\langle 1 / r\rangle$ & $\left\langle E_{k}\right\rangle$ & $P_{\Pi}$ & $m_{H}$ & $\langle 1 / r\rangle$ & $\left\langle E_{k}\right\rangle$ & $P_{\Pi}$ \\
\hline$\overline{H_{1}}$ & \multirow{4}{*}{1} & \multirow{2}{*}{$1^{--},(0,1,2)^{-+}$} & $\overline{4.15}$ & 0.42 & $\overline{0.16}$ & $\overline{0.82}$ & $\overline{7.48}$ & 0.46 & 0.13 & $\overline{0.83}$ & $\overline{10.79}$ & 0.53 & $\overline{0.09}$ & 0.86 \\
\hline$H_{1}^{\prime}$ & & & 4.51 & 0.34 & 0.34 & 0.87 & 7.76 & 0.38 & 0.27 & 0.87 & 10.98 & 0.47 & 0.19 & 0.87 \\
\hline $\mathrm{H}_{2}$ & & \multirow{2}{*}{$1^{++},(0,1,2)^{+-}$} & 4.28 & 0.28 & 0.24 & 1.00 & 7.58 & 0.31 & 0.19 & 1.00 & 10.84 & 0.37 & 0.13 & 1.00 \\
\hline$H_{2}^{\prime}$ & & & 4.67 & 0.25 & 0.42 & 1.00 & 7.89 & 0.28 & 0.34 & 1.00 & 11.06 & 0.34 & 0.23 & 1.00 \\
\hline $\mathrm{H}_{3}$ & 0 & $\left\{0^{++}, 1^{+-}\right\}$ & 4.59 & 0.32 & 0.32 & 0.00 & 7.85 & 0.37 & 0.27 & 0.00 & 11.06 & 0.46 & 0.19 & 0.00 \\
\hline $\mathrm{H}_{4}$ & 2 & $\begin{array}{l}\left\{2^{++},(1,2,3)^{+-}\right\} \\
\left\{2^{--},(1,2,3)^{-+}\right\}\end{array}$ & 4.37 & 0.28 & 0.27 & 0.83 & 7.65 & 0.31 & 0.22 & 0.84 & 10.90 & 0.37 & 0.15 & 0.87 \\
\hline$H_{6}$ & \multirow{2}{*}{3} & $\left\{3^{--},(2,3,4)^{-+}\right\}$ & 4.57 & 0.22 & 0.37 & 0.85 & 7.82 & 0.25 & 0.30 & 0.87 & 11.01 & 0.30 & 0.20 & 0.89 \\
\hline$H_{7}$ & & $\left\{3^{++},(2,3,4)^{+-}\right\}$ & 4.67 & 0.19 & 0.43 & 1.00 & 7.89 & 0.22 & 0.35 & 1.00 & 11.05 & 0.26 & 0.24 & 1.00 \\
\hline
\end{tabular}

$$
\left[-\frac{1}{m r^{2}} \partial_{r} r^{2} \partial_{r}+\frac{1}{m r^{2}}\left(\begin{array}{cc}
l(l+1)+2 & 2 \sqrt{l(l+1)} \\
2 \sqrt{l(l+1)} & l(l+1)
\end{array}\right)+\left(\begin{array}{cc}
E_{\Sigma}^{(0)} & 0 \\
0 & E_{\Pi}^{(0)}
\end{array}\right)\right]\left(\begin{array}{l}
\psi_{\Sigma}^{(N)} \\
\psi_{-\Pi}^{(N)}
\end{array}\right)=\mathcal{E}_{N}\left(\begin{array}{l}
\psi_{\Sigma}^{(N)} \\
\psi_{-\Pi}^{(N)}
\end{array}\right),
$$

and for the other we get the conventional radial Schrödinger equation

$$
\left[-\frac{1}{m r^{2}} \partial_{r} r^{2} \partial_{r}+\frac{l(l+1)}{m r^{2}}+E_{\Pi}^{(0)}\right] \psi_{+\Pi}^{(N)}=\mathcal{E}_{N} \psi_{+\Pi}^{(N)} .
$$

The two Schrödinger equations correspond to opposite parity solutions; also note that the first equation itself decouples for $l=0$ and only the solution for $\psi_{\Sigma}$ is physical

The results from the numerical solution of this equations is displayed in Table 1 . We label by $H_{1}$, $H_{2}$, etc the multiplets of hybrid states with same parity and angular momentum $l$ but different spin state. All states in a multiplet are mass degenerate in our approach since we do not consider spin dependent terms in our Hamiltonian.

\section{Comparisons}

In Ref. [15] the BO approximation was used to obtain the hybrid masses from the gluonic static energies computed on the lattice. They did not consider the hybrid potential mixing in the Schrödinger equation, which leads to the hybrid states appearing in terms of degenerate parity doublets. Considering the mixing terms results in the breaking of the degeneracy between the $H_{1}$ and $H_{2}$ multiplets as well as the $H_{4}$ and $H_{5}$ multiplets. We have plotted the results from [15] suitable for comparison with our results in Fig. 2 for charmonium hybrids. Comparing with the results from [15], we can see that the effect of introducing the mixing terms lowers the masses of the multiplets that have mixed contributions from the two hybrid static energies. The difference in masses for the $H_{2}$ and $H_{5}$ multiplets corresponds to an overall mass shift due to a different origin of energies for the potentials.

The spectrum of hybrids in the charmonium sector has been calculated by the Hadron Spectrum Collaboration [16] using unquenched lattice QCD. The calculations were done with unphysically pion mass of $\approx 400 \mathrm{MeV}$. The results from [16] are given with the $\eta_{c}$ mass subtracted and are not extrapolated to the continuum limit. The direct lattice results can be assigned to the pNRQCD multiplets, however, this assignment is ambiguous because some $J^{P C}$ quantum numbers appear more than once in the $H_{2}, H_{3}$, and $H_{4}$ multiplets. We assigns states to a specific multiplet based on the closeness in mass. 


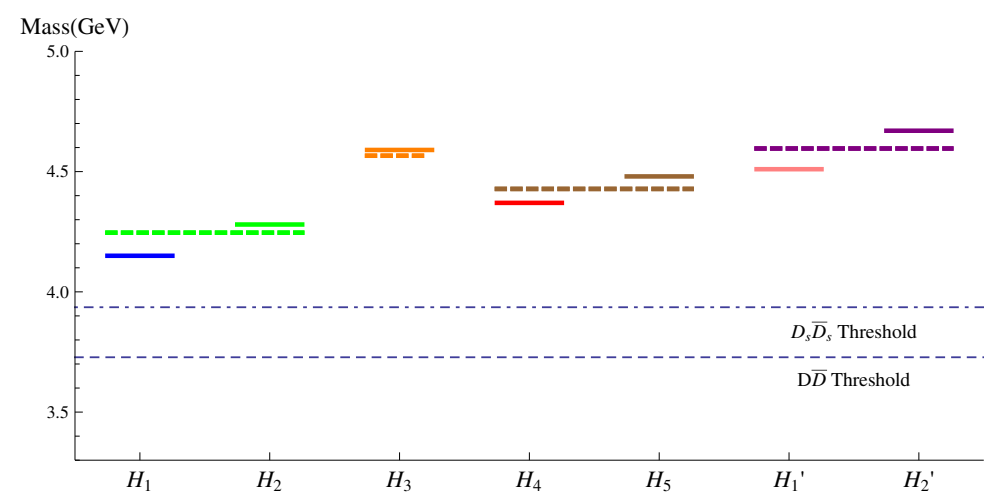

Figure 2. Comparison of the hybrid multiplet masses in the charmonium sector in Ref. [15] with the our results. Ref. [15] results correspond to the dashed lines, while the solid lines correspond to our results. The degeneracy of the masses of the $H_{1 / 2}$ and $H_{4 / 5}$ multiplets in Ref. [15] is broken by the introduction of the mixing terms in our approach.

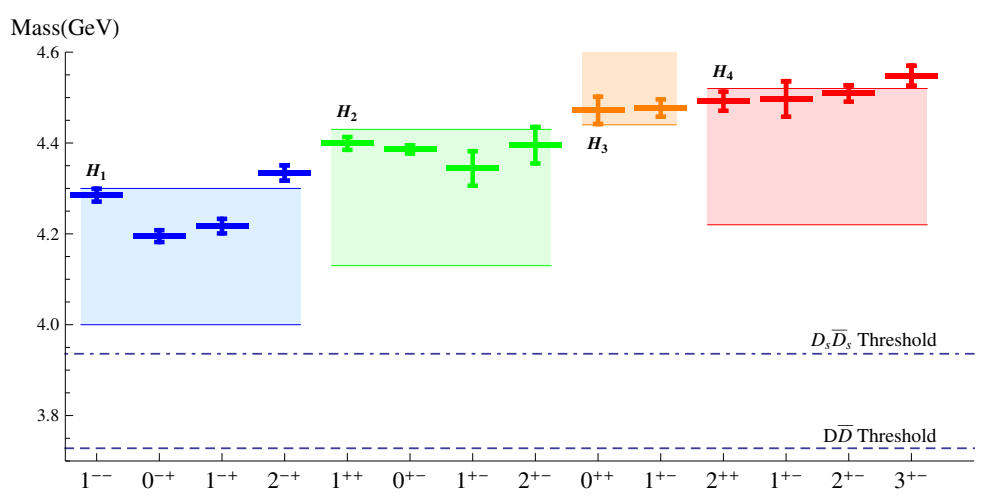

Figure 3. Comparison of the results from direct lattice computations of the masses for charmonium hybrids [16] with our results. The direct lattice mass predictions are plotted in solid lines with error bars corresponding to the mass uncertainties. Our results for the $H_{1}, H_{2}, H_{3}$, and $H_{4}$ multiplets have been plotted in error bands corresponding to the gluelump mass uncertainty of $\pm 0.15 \mathrm{GeV}$.

In Fig. 3 the results from [16] have been plotted (with the experimental value of $m_{\eta_{c}}=2.9837(7) \mathrm{GeV}$ added) together with our results. Looking at Fig. 3, the direct lattice calculation seems to support the result of our approach that the hybrid states appear in three distinct multiplets $\left(\mathrm{H}_{2}, \mathrm{H}_{3}\right.$, and $\left.\mathrm{H}_{4}\right)$ as compared to the constituent gluon picture, where they are assumed to form one supermultiplet together.

The candidates for quarkonium hybrids consists of the neutral heavy quark mesons above open flavor threshold. Most of the candidates have $1^{--}$or $0^{++} / 2^{++}$, since the main observation channels are production by $e^{+} e^{-}$or $\gamma \gamma$ annihilation, respectively, which constrains the $J^{P C}$ quantum numbers. It is important to keep in mind that the main source of uncertainty of our results is the uncertainty of the gluelump mass $\Lambda_{1^{+-}}^{R S}=0.87 \pm 0.15 \mathrm{GeV}$. We have plotted the candidate experimental states in Fig. 4, except for the single one corresponding to the bottomonium sector, overlaid onto our results with error 


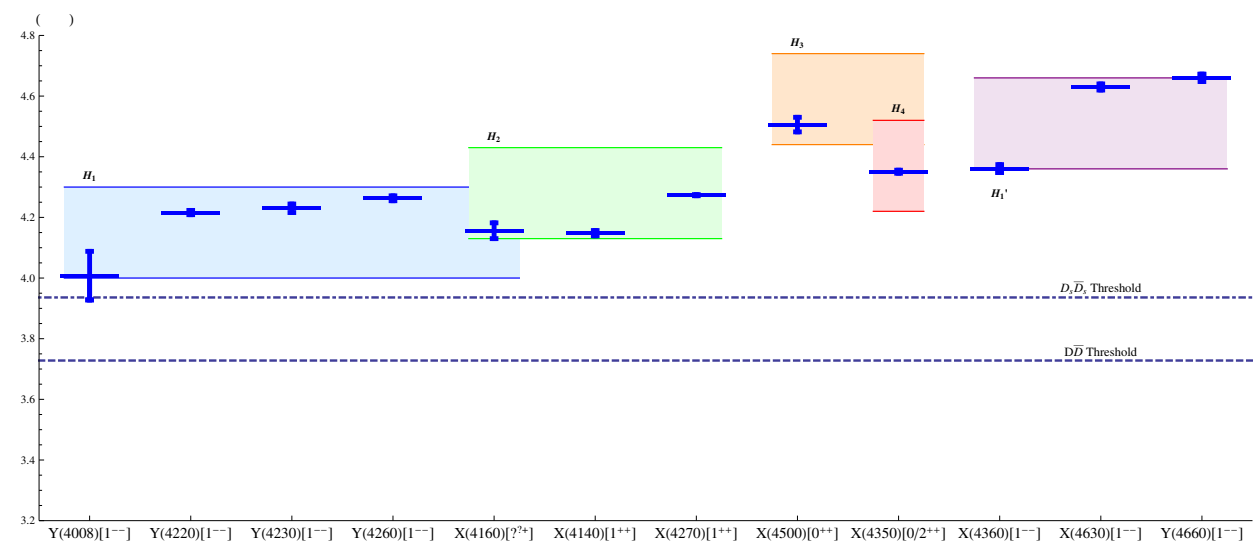

Figure 4. Comparison of the experimental candidate masses for the charmonium sector with our results using the $V^{(0.25)}$ potential. The experimental states are plotted in solid blue lines with error bars corresponding to the average of the lower and upper mass uncertainties. Our results for the $H_{1}, H_{2}, H_{4}$ and $H_{1}^{\prime}$ multiplets have been plotted in error bands corresponding to the gluelump mass uncertainty of $\pm 0.15 \mathrm{GeV}$.

bands corresponding to the uncertainty of the gluelump mass. Most of these candidate states decay to spin triplet charmonium but their $J^{P C}$ quantum numbers and mass fits spin singlet hybrid states, which in principle disfavors the hybrid interpretation. Nevertheless, there might be enough heavy quark spin symmetry violation to explain those decays. The main exception is the $Y(4220)$, which has been observed decaying to spin singlet quarkonium, which makes it a very good candidate for a charmonium hybrid.

\section{Conclusions}

We have reported [1] on the computation of the heavy hybrid masses using an EFT formulation that incorporates the energy gap between the gluonic and heavy-quark degrees of freedom. This EFT is constructed by a procedure analogous to the one used in the BO approximation for diatomic molecules. Starting from weakly-coupled pNRQCD we expand the octet sector in eigenstates of the leading order Hamiltonian, then the gluonic degrees of freedom at the $\Lambda_{Q C D}$ scale can be formally integrated out. The potential that appears in the EFT can be obtained by matching with NRQCD lattice calculations for the heavy-quark antiquark excited static energies. There are operators in the EFT, analogous to the nonadiabatic coupling in molecular physics, that mix static states which in the short distance are generated by the same gluelump. Since the potentials associated to the mixed states are quasi-degenerate in the short distance limit, we solve the coupled Schrödinger equations instead of using perturbation theory. The effect of the mixing terms is to break the mass degeneracy between opposite parity spin symmetry multiplets and specifically it has been found to lower the mass of the multiplets that get mixed contribution of different static energies. The same pattern of is observed in direct lattice calculations. A large set of masses for spin symmetry multiplets for $c \bar{c}, b \bar{c}$, and $b \bar{b}$ hybrids has been obtained for the $\Pi_{u}$ and $\Sigma_{u}^{-}$static energies. Mass gaps between multiplets are in good agreement with the spin averaged direct lattice computation values, but the absolute values are shifted. Several experimental candidates for charmonium hybrids and one for bottomonium hybrids have been found, being $Y(4220)$ the most promising. 


\section{References}

[1] M. Berwein, N. Brambilla, J. Tarrús Castellà, A. Vairo, Phys. Rev. D92, 114019 (2015), 1510.04299

[2] N. Brambilla et al., Eur. Phys. J. C71, 1534 (2011), 1010. 5827

[3] S.K. Choi et al. (Belle), Phys. Rev. Lett. 91, 262001 (2003), hep-ex/0309032

[4] B. Aubert et al. (BaBar), Phys. Rev. Lett. 95, 142001 (2005), hep-ex/0506081

[5] R. Aaij et al. (LHCb), Phys. Rev. Lett. 115, 072001 (2015), 1507.03414

[6] G.T. Bodwin, E. Braaten, E. Eichten, S.L. Olsen, T.K. Pedlar, J. Russ, Quarkonium at the Frontiers of High Energy Physics: A Snowmass White Paper, in Proceedings, Community Summer Study 2013: Snowmass on the Mississippi (CSS2013): Minneapolis, MN, USA, July 29-August 6, 2013 (2013), 1307.7425, https://inspirehep.net/record/1245049/files/arXiv: 1307.7425.pdf

[7] N. Brambilla et al., Eur. Phys. J. C74, 2981 (2014), 1404 . 3723

[8] A. Pineda, J. Soto, Nucl. Phys. Proc. Suppl. 64, 428 (1998), hep-ph/9707481

[9] N. Brambilla, A. Pineda, J. Soto, A. Vairo, Nucl. Phys. B566, 275 (2000), hep-ph/9907240

[10] N. Brambilla, A. Pineda, J. Soto, A. Vairo, Phys. Rev. D63, 014023 (2001), hep-ph/0002250

[11] K.J. Juge, J. Kuti, C.J. Morningstar, Nucl. Phys. Proc. Suppl. 63, 326 (1998), hep-lat/9709131

[12] K.J. Juge, J. Kuti, C. Morningstar, Phys. Rev. Lett. 90, 161601 (2003), hep-lat/0207004

[13] G.S. Bali, A. Pineda, Phys. Rev. D69, 094001 (2004), hep-ph/0310130

[14] M. Foster, C. Michael (UKQCD), Phys. Rev. D59, 094509 (1999), hep-lat/9811010

[15] E. Braaten, C. Langmack, D.H. Smith, Phys. Rev. D90, 014044 (2014), 1402 . 0438

[16] L. Liu, G. Moir, M. Peardon, S.M. Ryan, C.E. Thomas, P. Vilaseca, J.J. Dudek, R.G. Edwards, B. Joo, D.G. Richards (Hadron Spectrum), JHEP 07, 126 (2012), 1204 . 5425 